\title{
Analyse de la distribution des malacocénoses de 23 lacs français
}

\author{
J. Mouthon 1
}

Mots clés : Mollusques, lacs, analyses multivariées, matière organique.

L'application de techniques d'analyses multivariées à l'inventaire malacologique de 23 lacs d'altitude moyenne montre que, suivant l'axe l exprimant " l'effet profondeur », s'effectue une simplification verticale des peuplements. L'axe 2 semble essentiellement traduire une modification des malacocénoses sous l'effet d'une accumulation croissante des matières organiques dont l'importance sur la structure et la distribution des peuplements est ainsi mise en évidence.

\section{Distribution of molluse communities in 23 French lakes}

Keywords : Mollusca, lakes, multivariates analysis, organic matter.

The freshwater Mollusca populations of 23 French lakes situated between 478 and $1152 \mathrm{~m}$ height are analysed by means of a factorial correspondence analysis. The first axis represents a " depth effect " factor characterized by a vertical simplification of mollusc communities. The second axis which separates mollusc populations of the littoral zones suggests that the organic matter is the chief factor in the species distribution.

\section{Introduction}

Les monographies écologiques consacrées aux écosystèmes lacustres dans lesquelles les peuplements de Mollusques sont décrits d'une manière plus ou moins détaillée, les Sphaeriidae étant trop fréquemment négligés, sont relativement nombreuses.

En revanche, on ne retrouve pas dans la littérature de travaux synthétiques confrontant les relevés malacologiques d'une série de milieux lacustres.

Ce sont les résultats d'une application d'analyses multivariées, aux peuplements de 23 plans d'eau dont les principales caractéristiques morphologiques sont rassemblées dans le tableau 1 , que nous présentons ici.

Parmi ces lacs situés dans une gamme d'altitude moyenne comprise entre 478 et 1152 mètres, 4 appartiennent à des régions cristallophyliennes (Vosges et Massif-Central), les 19 autres sont localisés dans le massif calcaire du Jura franc-comtois.

1. Laboratoire d'Hydrobiologie du CEMAGREF - 3, quai Chauveau 69336 Lyon Cédex 09.
Leurs malacocénoses ont généralement été étudiées dans le cadre de diagnoses écologiques à caractère synt hétique (Lab. Hydrobiol. Univ. Fr.-Comté, 1979 ; CTGREF, 1979 ; EPR Fr.-Comté 1982, 1984, $1985,1986 a, b, 1987 \mathrm{a}, \mathrm{b}$ ou ont fait l'objet de recherches spécifiques (Mouthon 1981). D'autres caractéristiques concernant l'histoire, la morphologie, la physico-chimie et la biologie de ces lacs figurent également dans les travaux de Delebecque (1898), Magnin (1904), Hubault (1947), Larue (1958), Lafont \& Juget (1981), Lafont (1982), Campy (1982), Verneaux et al. $(1987,1988) \ldots$

\section{Matériel et méthodes}

L'hétérogénéité des habitats rencontrés dans la zone littorale de la plupart de ces lacs, notamment la présence de ceintures végétales plus ou moins bien développées, nous a conduit à réaliser un échantillonnage spécifique pour les profondeurs comprises entre 0 et $1 \mathrm{~m}$.

Les Mollusques de cette zone ont été récoltés à l'aide d'un troubleau à base rectangulaire $(25 \times 18$ $\mathrm{cm})$. L'échantillonnage réalisé sur toute la largeur 
Tableau 1. - Liste et caractéristiques générales des milieux prospectés (d'après Delebecque 1898 et Magnin 1904). (1) en hautes eaux.

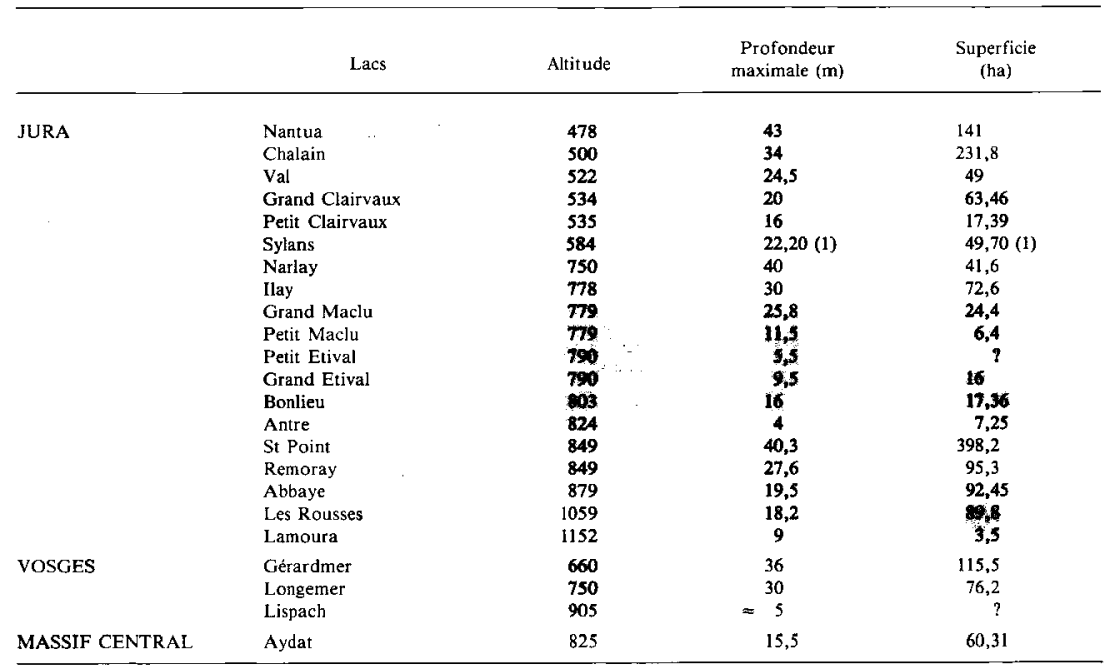

de la zone rivulaire représente une surface totale de 1,2 , parfois $3 \mathrm{~m}^{2}$ par station. Le nombre de ces stations pouvant aller de 3 à 13 suivant la taille des lacs et la diversité de leurs faciès littoraux. Seul l'effectif moyen des espèces récoltées a été retenu dans cette étude.

Les zones profondes ont été prospectées à l'aide d'une benne de Friedinger (surface $=350 \mathrm{~cm}^{2}$ ), trois prélèvements étant effectués à chaque point d'échantillonnage. Ceux-ci sont répartis sur deux, trois ou quatre transects, choisis en fonction des caractéristiques morphologiques de chaque plan d'eau, aux profondeurs de $2-3,5,10,20,25,30,35,40 \mathrm{~m}$. pour les milieux les plus profonds. Seul l'effectif moyen des Mollusques, à chaque point a été retenu.

Afin de pouvoir comparer les effectifs des peuplements littoraux à ceux récoltés aux différentes profondeurs, la densité des espèces, sur chaque station est rapportée au $\mathrm{m}^{2}$.
Les échantillons furent tamisés à $630{ }_{\mu} \mathrm{m}$, puis triés et déterminés à l'aide d'une loupe binoculaire.

Les données ainsi obtenues ont été rangées dans une matrice où figurent en ligne, les différentes profondeurs prospectées pour chaque lac et en colonne, les espèces recensées. A l'intersection d'une ligne et d'une colonne apparaît la densité au $\mathrm{m}^{2}$ de l'espèce spn, à la profondeur Pn.

Les différents programmes d'analyse des données appliqués dans ce travail sont l'analyse factorielle des correspondances et la classification ascendante hiérarchique dont on trouvera les fondements mathématiques dans les ouvrages de Benzecri $(1976 a, b)$, ainsi que différentes techniques d'aides à l'interprétation. Ces logiciels sont tous extraits de la bibliothéque ADDAD. La distance entre individus et le critère d'agrégation utilisés sont respectivement le $x^{2}$, et le moment centré d'ordre 2 . Les traitements ont été effectués sur un ordinateur de type VAX. 


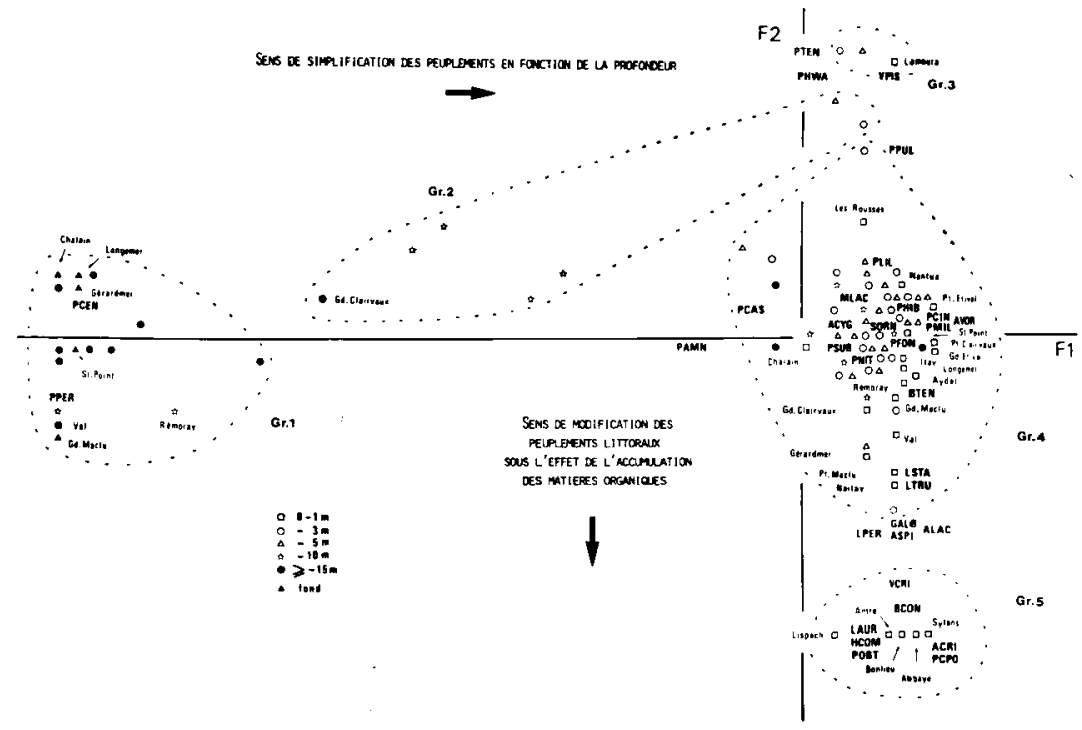

Fig. 1. - Distribution des stations et des espèces dans le plan des axes 1 et 2 (le nom du lac figure au niveau de sa station littorale $(0-1 \mathrm{~m})$ et profonde, si elle héberge des peuplements différenciés).

\section{Organisation des stations et des espèces dans l'espace factoriel}

L'application de l'analyse factorielle des correspondances à la matrice des données brutes, nous a fourni une image de la distribution des points d'échantillonnage et des espèces dans le plan des deux premiers axes, représentant $31,9 \%$ du taux d'inertie totale du nuage, respectivement $20,8 \%$ pour F1 et $11,1 \%$ pour F2 (fig. 1).

\subsection{Les groupements des stations}

Un programme de classification ascendante hiérarchique appliquée aux points d'échantillonnage a permis un regroupement des stations à l'intérieur de cinq noyaux.

\section{- Groupement 1}

Il renferme toutes les stations profondes des lacs au niveau desquels les 2 Bivalves inféodés à cette zone : Pisidium conventus et $\boldsymbol{P}$. personatum, ou seulement ce dernier dans le cas de la station $-10 \mathrm{~m}$ du lac de Rémoray, sont dominants.

- Groupement 2

C'est un groupement intermédiaire entre zone profonde et zone littorale. On y trouve toutes les stations sublittorales des lacs de Chalain, Longemer, Gérardmer et du Grand Lac de Clairvaux qui abritent encore les deux espèces de Bivalves mentionnées ci-dessus, mais également les profondeurs 3 et $5 \mathrm{~m}$. du Petit Lac de Clairvaux, où prolifère Pisidium tenuilineatum, Bivalve essentiellement littoral dans les autres lacs étudiés. 


\section{- Groupement 3}

Ce noyau associe les stations représentant la zone littorale du lac de Lamoura et les points - 3 et $5 \mathrm{~m}$. du lac de Nantua. Elles ont en commun le même taxon dominant : Valvata piscinalis et des peuplements peu diversifiés avec respectivement 9 espèces pour la première et 5 pour les deux autres, largement perturbées par des rejets domestiques anciens (Vivier \& Serruya, 1966).

La prolifération d'un dense tapis de Characées envahissant la totalité de la zone littorale du lac de Lamoura, limitant fortement l'installation de ceintures végétales différenciées, explique la structure particulière des malacocénoses littorales de ce lac, le plus élevé du Jura (alt. $1152 \mathrm{~m}$ ).

\section{- Groupement 4}

Il réunit les points de prélèvements littoraux de la plupart des milieux, ainsi que les stations sublittorales et profondes des lacs dans lesquels il n'existe pas de peuplements profonds différenciés, soit à cause de conditions physico-chimiques défavorables, notamment un déficit important de l'hypolimnion en oxygène dissous en dehors des périodes de circulation, (cf. Nantua, Narlay, Ilay...), soit parce que la profondeur du milieu est notablement insuffisante pour permettre leur développement (Antre, Etival...). On y trouve également les stations profondes, au niveau desquelles les espèces caractéristiques de cette zone ne sont plus les éléments dominants de la malacocénose, comme les points $-15 \mathrm{~m}$ du lac de Rémoray et $-10,-25 \mathrm{~m}$ du lac de St Point. L'existence d'un vecteur fluviatile traversant ce plan

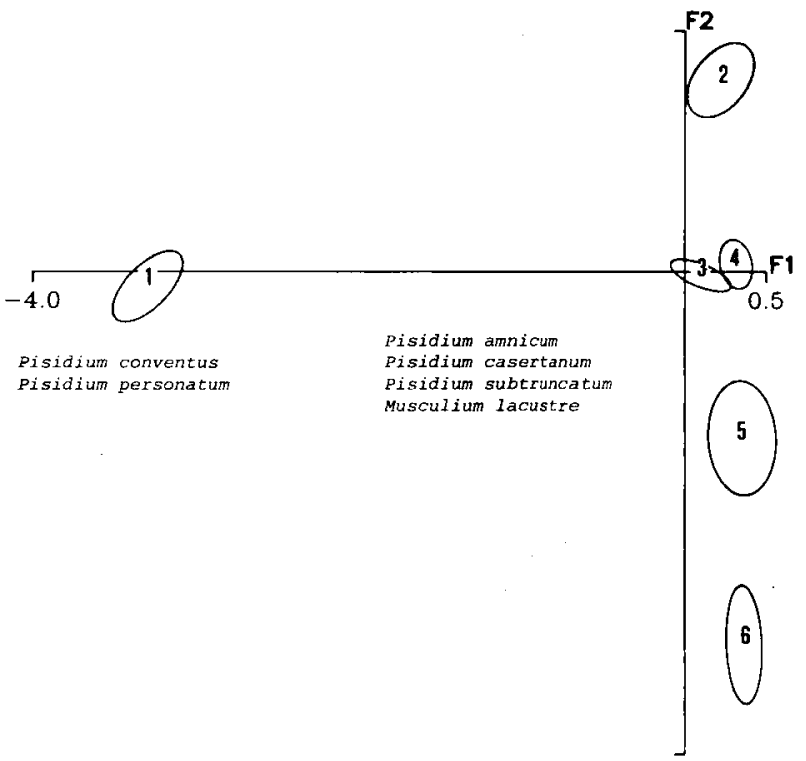

Valvata piscinalis

Pisidium pulchellum Pisidium tenuilineatum Pisidium henslowanum

Bithynia tentaculata Physa fontinalis Lymnaea palustris anisus vortex Planorbis carinatus Anodonta cygnea Pisidiun hibernicum Pisidium lijjeborgii Pisidium milium Pisidium nitidum Sphaerium corneum

Lymnaea stagnalis Valvata cristata Lymnaea peregra Lymnaea truncatula Anisus spirorbis Bathyomphalus contortus Gyraulus albus Armiger crista Hippeutis complanata Acroloxus lacustris

Lymnaea auricularia Pisidium casertanum F.ponderosa

Pisidium obtusale

Fig. 2. - Situation dans le plan des axes F1, F2 des ellipses d'intertie représentant les groupements d'espèces. 
d'eau est sans doute une des causes principales de la présence d'espèces littorales comme Pisidium hibernicum et $\boldsymbol{P}$. nitidum à une telle profondeur (Verneaux et al., 1988).

\section{- Groupement 5}

On y trouve les stations littorales riches en matières organiques des lacs de Bonlieu, l'Abbaye, Antre et Lispach ou présentant des faciès d'atterrissement bien développés comme le lac de Sylans.

\subsection{Les groupements d'espèces}

A partir de l'arbre de la classification une partition en 6 groupements a été effectuée, leur distribution dans l'espace factoriel des axes 1 et 2 apparait sous forme d'ellipses d'inertie sur la figure 2.

\section{- Groupement 1}

Il est constitué de deux Bivalves colonisant les zones profondes des systèmes lacustres médioeuropéens Pisidium conventus et $P$. personatum et représente l'essentiel de la contribution totale des colonnes à l'axe 1, soit $913 \%$ (Tableau II).

\section{- Groupement 2}

Il renferme 3 Lamellibranches plutôt littoraux, mais relativement peu fréquents parmi les lacs

Tableau II. - Codes, qualité de la représentation (COR) et contribution relative (CTR) des espèces à l'inertie des axes I, 2 et 3.

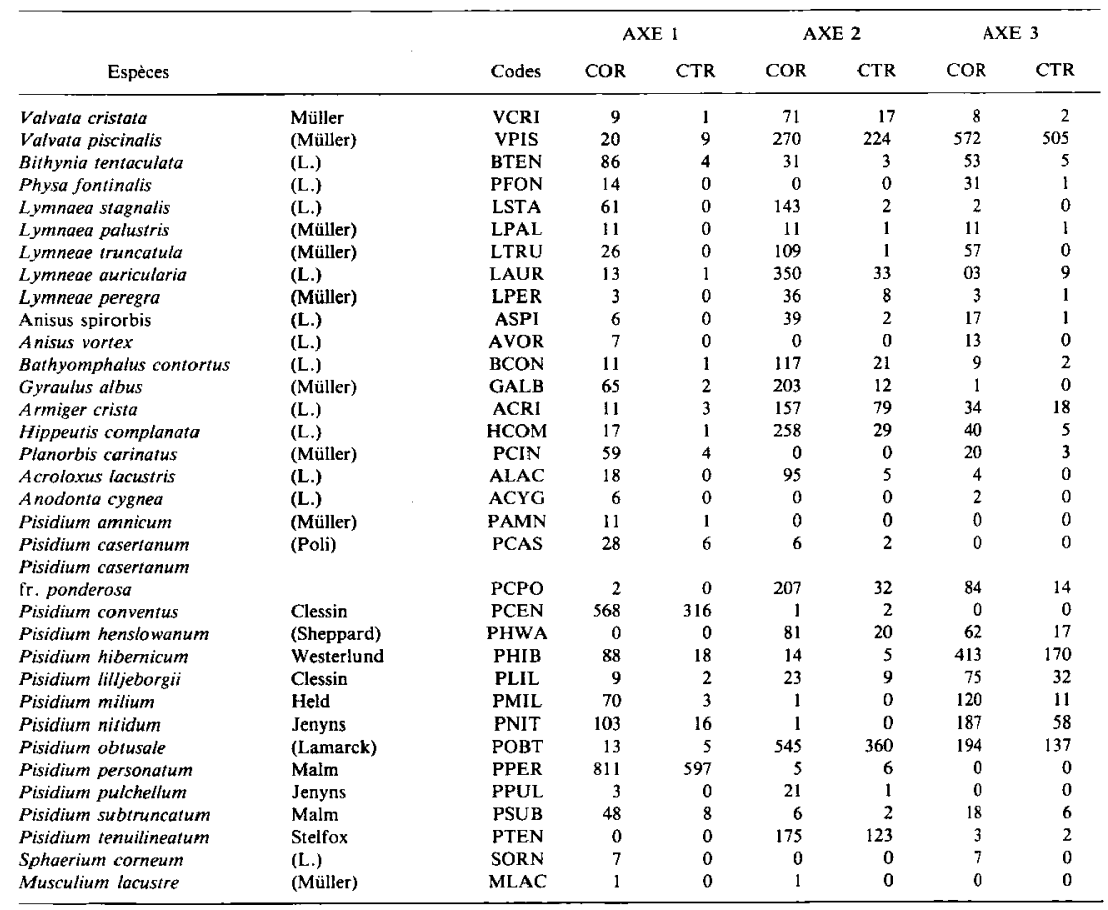


étudiés, et 1 Gastéropode Valvata piscinalis plus eurybathe (cf. Mouthon 1986). Associès à Pisidium tenuilineatum, ces deux espèces représentent $347 \%$ de la contribution à l'axe 2 .

\section{- Groupement 3}

Il comprend 4 espèces, toutes des Bivalves. Parmi celles-ci, seule Musculium lacustre est strictement littorale, elle colonise les faciès vaseux riches en matières organiques, généralement en compagnie de Pisidium subtruncatum. Cette dernière et les deux autres mollusques appartenant à ce noyau ont des amplitudes bathymétriques beaucoup plus fortes puisqu'ils atteignent et parfois même dépassent sensiblement la zone sublittorale.

\section{- Groupement 4}

Ce noyau proche de l'origine des axes comme le précédent, compte 11 espèces dont 5 Gastéropodes, il constitue avec le groupement 3 le peuplement typique des zones littorales et sublittorales de la plupart des lacs étudiés.

- Groupement 5

Il réunit 10 espèces, essentiellement des Gastéropodes. Toutes, à l'exception de Lymnaea peregra que l'on trouve dans la zone profonde de certains grands lacs (Forel 1885, Zschokke 1979, Alsterberg 1930), sont strictement inféodées aux zones de bordure riches en macrophytes et débris organiques. Certaines d'entre elles comme Lymnaea iruncatula, Anisus spirorbis, Valvata cristata... peuvent même supporter des périodes d'assèchement plus ou moins prolongées (Boycott 1936, Marazanof 1970, YacineKassab 1979).

\section{- Groupement 6}

Cette curieuse association d'espèces est caractéristique du lac de Sylans. Du fait de l'importance du marnage qui affecte ce lac chaque été, on trouve au niveau de ses bordures deux types de peuplements. En hautes eaux, c'est-à-dire pendant près des $2 / 3$ de l'année, c'est Pisidium obtusale, Bivalve qui supporte bien la dessication (Meier-Brook 1975) qui domine les peuplements littoraux, en compagnie de quelques spécimens de Lymnaea auricularia et de Planorbis carinatus. En revanche à l'étiage, lorsque la zone eulittorale est asséchée, c'est $L$. auricularia qui prolifère dans les herbiers à nénuphars, tandis qu'au niveau des sédiments on ne trouve que de rares exemplaires de Pisidium casertanum, mais uniquement sous sa forme ponderosa. Il est intéressant de signaler que parmi les lacs étudiés, c'est le seul qui héberge cette forme particulière que l'on trouve généralement surtout dans les grands lacs de plaine comme le Léman, le Bourget, Neuchatel... (Favre $1927,1940,1941)$. Ce groupement associe donc une espèce caractéristique des faciès eulittoraux : Pisidium obfusale qui représente $360 \%$ de la contribution à l'axe 2, à deux espèces littorales $L$. auricularia et $P$. casertanum forme ponderosa.

\subsection{Structure des peuplements malacologiques des lacs}

Elle est obtenue en joignant les points d'échantillonnage de chaque lac, de sa zone profonde vers sa zone littorale. A partir de l'examen de cette structure il est possible de classer ces milieux à l'intérieur de 3 groupes ou sous-groupes. Les exemples choisis pour illustrer ces différents types de distribution des peuplements apparaissent sur la figure 3.

3.3.1. Lacs avec des peuplements profonds différenciés

Ce sont les lacs de Chalain, Grand-Maclu, SaintPoint, Val, Rémoray, Longemer, Gérardmer et Grand-Clairvaux. Dans leur zone profonde ou seulement dans une partie de celle-ci, lorsque le déficit en 02 dissous de leur hypolimnion trop important, devient limitant pour ces organismes (Thieneman 1913, Valle 1927, Meier-Brook 1963), on récolte Pisidium conventus et $P$. personatum, ou seulement ce dernier dans le lac de Rémoray.

Lorsque ces deux espèces cohabitent avec d'autres Mollusques plus littoraux tels que Pisidium casertanum, $P$. subtruncatum... la position de la station concernée se trouvera d'autant plus proche de l'axe 2 que la représentation des Bivalves d'eaux profondes sera faible par rapport aux autres Mollusques. C'est ce que l'on observe pour la station profonde du Grand Clairvaux, des points $-15 \mathrm{~m}$. du lac de Rémoray et $-10,-25 \mathrm{~m}$. du lac de St Point (fig. 1 et 3 ).

3.3.2. Lacs sans peuplements profonds différenciés

Ce sont tous les autres milieux étudiés. Parci ceuxci on trouve évidemment les milieux dont la profondeur est inférieure à $10 \mathrm{~m}$. : lacs de Lispach, Antre, Petit et Grand-Etival, mais également un ensemble de plans d'eau plus profonds comme Nantua, Narlay, Ilay (cf. Tableau I).

Deux sous-groupes peuvent être distingués parmi ces lacs. 


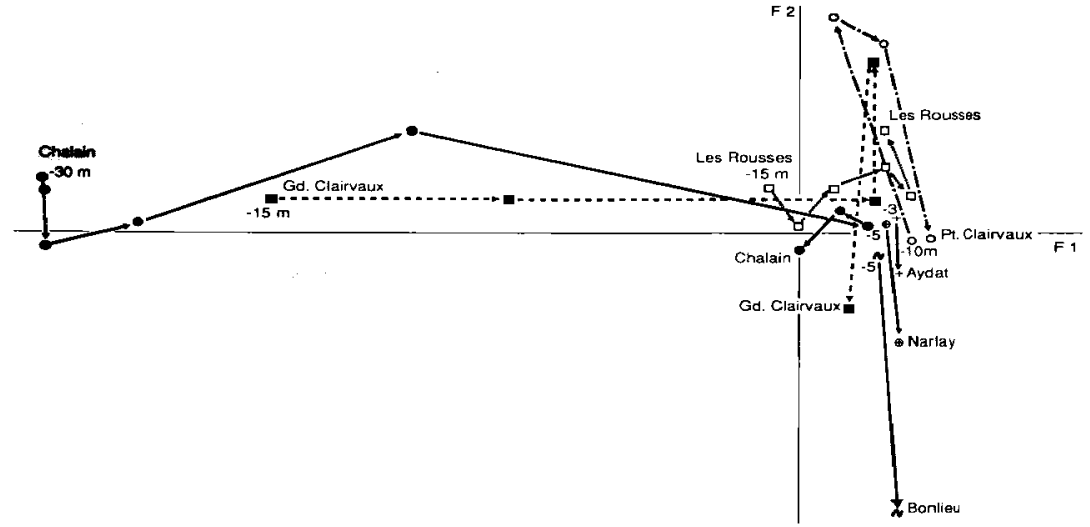

Fig. 3. - Structure malacologique de quelques lacs.

- Dans le premier, on trouve les lacs de Sylans, Lispach, l'Abbaye, Antre et Bonlieu. Leurs stations rivulaires riches en matières organiques appartiennent au groupement 5 (fig. 1). Elles sont essentiellement peuplées d'espèces peu exigeantes et strictement littorales. En revanche leurs stations plus profondes $-3,-5 \mathrm{~m}$... qui hébergent des peuplements très appauvris dominés par Pisidium subtruncatum, plus eurytope et eurybathe, appartiennent au groupement 4 .

- Dans le second sous-groupe figurent les milieux non encore cités. Les espèces qui colonisent leur zone sublittorale vivent également dans leur zone littorale ; de ce fait, la majorité de ces stations appartiennent au groupement 4. Seuls quelques points où des espèces peu fréquentes sont bien représentées se trouvent dans le groupement 3 : Lamoura 0-1 m., Nantua -3 et $-5 \mathrm{~m}$. ou dans le groupement 2 : Petit-Clairvaux -3 et $-5 \mathrm{~m}$.

La confrontation de cette classification à celle proposée par Verneaux et al. (1987) à partir de la teneur en carbone organique et du rapport minéral : $\mathrm{Ca} / \mathrm{Si}$ des sédiments profonds de 10 lacs, parmi les 23 retenus dans cette étude, montre que seuls deux types de lacs hébergent des peuplements différenciés dans leur zones profondes (groupe I).
Il s'agit des milieux dont les sédiments relativement pauvres en carbone organique $(\mathrm{C}<8 \% \mathrm{~m} . \mathrm{s}$.) sont surtout de nature détritique comme Chalain, Saint-Point et le Grand-Maclu, avec des ratios $\mathrm{Ca} / \mathrm{Si}$ compris entre 5 et 7 ou biochimique tel le GrandClairvaux qui, avec un rapport $\mathrm{Ca} / \mathrm{Si}>12$ est considéré comme associé au pôle carbonaté par ces auteurs.

En revanche, lorsque les concentrations en carbone organique des sédiments profonds augmentent $(8 \%<\mathrm{C}<15 \%$ m.s.), alors que les valeurs du rapport $\mathrm{Ca} / \mathrm{Si}$ demeurent très variables $(1<\mathrm{Ca} / \mathrm{Si}$ $<8$ ), lacs d'Etival, des Rousses et d'llay, puis sont directement associées au pôle organique $(15 \%<$ $\mathrm{C}<20 \%$ et $0<\mathrm{Ca} / \mathrm{Si}<3$ ) comme dans les lacs de Bonlieu, Petit-Maclu et l'Abbaye ; les zones profondes et parfois sublittorales de ces milieux sont dépourvues de Mollusques (groupe II). Ceci montrerait le rôle important de la matière organique dans la distribution des malacocénoses lacustres. Toutefois une analyse statistique permettant d'étudier plus en détail les relations existant entre les peuplements et différents paramètres physico-chimiques de la masse d'eau et des sédiments profonds s'avère nécessaire et sera envisagée ultérieurement ; d'autres plans d'eau étant actuellement à l'étude. 


\section{Conclusion}

L'application des techniques d'analyse de données à l'inventaire malacologique de 23 lacs d'altitude moyenne, nous a permis de présenter une organisation des stations et des espèces dans l'espace des 2 premiers axes.

A partir de cette structure, il est possible de dresser la composition malacologique type d'un lac théorique d'altitude moyenne, dont l'image la plus proche nous est fournie par les peuplements du lac St. Point, comprenant 29 espèces sur les 33 recensées dans les milieux étudiés.

Les stations d'eau profonde colonisées par des peuplements différenciés s'organisent le long de l'axe 1 exprimant « l'effet profondeur ». En revanche, les points $d$ 'échantillonnage où dominent les espèces littorales sont répartis le long de l'axe 2. Plus complexe, la signification de ce dernier semble essentiellement traduire une modification progressive de la structure des peuplements, sous l'effet d'une accumulation croissante des matières organiques.

L'examen de la structure malacologique de chacun des milieux a montré qu'une simplification verticale des peuplements s'effectue suivant l'axe 1 , se traduisant par une disparition des Mollusques d'eaux profondes parallèlement à une réduction croissante de l'amplitude bathymétrique des espèces (cf. Mouthon 1986).

Par ailleurs, la confrontation de la classification malacologique des lacs présentée ici aux caractères généraux des sédiments profonds de 10 d'entre eux (Verneaux et al., 1987) a mis en évidence l'importance du rôle joué par les matières organiques dans la structure de la distribution des malacocénoses littorales, sublittorales et profondes en milieu lacustre.

Nous nous proposons, ultérieurement, de définir plus précisément les relations existant entre malacocénoses et paramètres physico-chimiques des sédiments et de la masse d'eau.

\section{Remerciements}

L'auteur remercie vivement Messieurs F. Trocherie (Ingénieur au CEMAGREF) pour son aide particulièrement appréciée dans l'analyse des données et $\boldsymbol{J}$. Verneaux (Professeur à l'Université de Besançon) dont les précieux conseils ont guidé la rédaction de ce texte, ainsi que Madame Taillole qui en a assuré la dactylographie.

\section{Travaux cités}

Alsterberg (G.). 1930. - Wichtige Züge in der Biologie der Süsswassergastropoden. Lund (A-B. Gleerlupska Unit. Bokhandeln) : $130 \mathrm{p}$.

Benzecri (J.P.). 1976a. - L'analyse des données. 1. La Taxinomie. Dunod ed. Paris, $631 \mathrm{p}$.

Benzecri (J.P.). 1976b. - L'analyse des données. 2. L'analyse des correspondances. Dunod ed. Paris, $616 \mathrm{p}$.

Boycott (A.E.). 1936. - The habitats of the fresh-water Mollusca in Britain. J. anim. Ecol., S : 116-186.

Campy (M.). 1982. - Le Quaternaire Franc-Comtois. Essai chronologique et paléo climatique : Thèse Doc. es Sciences, Besançon, 528 p.

CTGREFI. 1979. - Etude écologique des lacs des Vosges: Gerrardmer et Longemer. Etude $n^{\circ} 44: 79 \mathrm{p}$.

Delebecque (A.). 1898 , - Les lacs français. Chamerot et Renovard eds, Paris, $436 \mathrm{p}$.

E.P.R. ${ }^{2}$ Fr. Comté. 1982. - Le lac de l'Abbaye (Massif du Jura). Etude écologique et biologique : $61 \mathrm{p}$.

E.P.R. Fr. Comté. 1984. - Les lacs de Clairvaux. Monographies écologiques : $113 \mathrm{p}$.

E.P.R. Fr. Comté. 1985. - Les lacs Maciu (Jura). Monographies écologiques : $130 \mathrm{p}$.

E.P.R. Fr. Comte. 1986a. - Le lac de Chalain (Jura). Etude écologique : $116 \mathrm{p}$.

E.P.R. Fr. Comté. 1986b. - Le lac d'llay (Jura). Eude écologique : $95 \mathrm{p}$.

E.P.R. Fr. Comté. 1987a. - Les lacs d'Etival (Jura) Diagnose : $87 \mathrm{p}$.

E.P.R. Fr. Comté. 1987b. - Le lac de Bonlieu (Jura). Diagnose : $100 \mathrm{p}$.

Favre (J.). 1927. - Les mollusques post-glaciaires et actuels du bassin de Genève. Mém. Soc. Phys. et Hist. nat. Genève, 40, $3: 171-430$.

Favre (J.). 1940. - La faune malacologique post-glaciaire et actuelle du lac du Bourget Ann. Ecole Nat. Eaux et Forêts, 7 (2) : 294-444.

Favre (I.). 1941. - Les Pisidium du canton de Neuchatel. Soc. Neuch. Sci. Nal., 66:57-112.

Forel (F.A.). 1885. - La faune profonde des lacs suisses. Nouveaux mémoires de la Soc, helv, de Sci. Nat.

Hubault (E). 1947. - Etudes thermiques, chimiques et biologiques des eaux des lacs de l'est de la France (Vosges, Jura, Alpes de Savoie). Annls. Ec. nat. Eaux Forêts, 10 (2) : $116-260$.

Laboratoire d'hydrobiologie et d'hydroécologie. 1979. - Etude ecologique des lacs de Saint-Point et de Rémoray. Univ. Fr. Comté éd., 114 p.

Lafont (M.) \& Juget (J.). 1981. - Les Oligochètes de quelques lacs jurassiens et leur utilisation pour apprécier l'état biologique des sédiments profonds. Ann. Sci. Univ. Fr. Comté, Biol. Anim. 4 (2): 47-57.

Lafont (M.). 1982. - Les Oligochètes des lacs de Gèrardmer et de Longemer (Département des Vosges, France), Sciences de l'ear, 1 (1) : 21-30.

Larue (R.). 1958. - Contribution à la connaissance limnologique du lac d'Aydat. Rev. Sci. nat. Auvergne, $24: 1-40$.

1. Centre Technique du Génie Rural des Eaux et des Forēts.

2. Etablissement Public Régional. 
Magnin (A.). 1904. - La végétation des lacs du Jura. Klinck. sieck ed. Paris, $426 \mathrm{p}$.

Marazanof (F.). 1970. - Contribution à l'étude ecologique des mollusques des eaux douces et saumâtres de Camargue. IL. Anists rotundalus (Poiret, 1801) Annis. Limnol. 6 (2) : 191-213.

Meier-Brook (C.), 1963, - Über die Mollusken der Hochschwarzwald-und Hochvogesen Gewässer. Arch. Hydrobiol. Suppl. $28(5): 1-46$.

Meier-Brook (C.). 1975. - Der ökologische Indikatorwert mitteleuropäischer Pisidium Arten (Mollusca, Eulamellibranchiata). Eiszeilaler $u$. Gegenwart. $26: 190-195$.

Mouthon (J.). 1981. - Les Mollusques des lacs de l'Abbaye et des Rousses (Massif du Jura). Ann. Sci. Univ. Besançon, 4 (2) : 1.35 .

Mouthon (J.). 1986. - Principes généraux pour une méthode d'appréciation de la qualité globale des sédiments lacustres à l'aide d'une analyse simplifiée des malacocénoses. $A n n l s$ Limnol. 22 (3) : 209-217.

Thienemann (A.), 1913. - Der Zusammenhang zwischen dem Sauerst offgehalt des Tiefenwassers und der Zusammensetzung der Tierfauna unserer Seen. Int. Revue ges. Hydrobiol. Hydrogr, $6: 243-249$.
Valle (K.S.). 1927. - Ökologische - limnologische Untersuchungen über die Boden und Tiefenfauna in einigen Seen nördliche vom Ladoga-see. Acta zool. fenn. $4: 1-231$.

Verneaux (J.), Vidonne (A.) \& Guyard (A.). 1987. - Caractères généraux des sédiments de 10 lacs jurassiens. Sciences de l'eau, $6: 107-128$

Verneaux (I.), Guyard (A.), Joly (D.) \& Vidonne(A.). 1988. Etude physico-chimique des sédiments du lac de St-Point (Massif du Jura) : cartographie thématique. Annls. Limnol. 24 (1) : 15-29.

Vivier (P.) \& Serruya (S.). 1966. - La pollution organique des lacs de Nantua. Eau : 167-171.

Yacine-Kassab (M.). 1979. - Etude écologique des Mollusques gastéropodes d'eau douce de quelques milieux aqualiques peu profond's de la vallé du Rhône en amont de Lyon. Thèse Doc. ìs Sciences, Grenoble, 2 vol. $247 \mathrm{p}$. et $103 \mathrm{p}$.

Zschokke (F.). 1911. - Die Tiefseefauna der Seen Mitteleuropas. Int. Revue ges. Hydrobiol. Hydrog., 4 : 1.246. 